\title{
Comparison of amniotic fluid volumetry between fetal sonography and MRI - Correlation to MR diffusion parameters of the fetal kidney
} \author{
Schlembach $^{5}$, Uwe Schneider ${ }^{3}$ and Hans-Joachim Mentzel ${ }^{1}$ \\ ${ }^{1}$ Institute of Diagnostic and Interventional Radiology, Section of Pediatric Radiology, University Hospital Jena, Germany \\ ${ }^{2}$ Institute of Diagnostic and Interventional Radiolgy, Medical Physics Group, University Hospital Jena, Germany \\ ${ }^{3}$ Department of Obstetrics, University Hospital Jena, Germany \\ ${ }^{4}$ Department of Pediatrics, Section of Pediatric Nephrology, University Hospital Jena, Germany \\ ${ }^{5}$ Clinic of Obstetrics, Vivantes Hospital Berlin-Neukoelln, Germany
}

Elena Moschos ${ }^{1 *}$, Daniel Güllmar², Anja Fiedler ${ }^{3}$, Ulrike John $^{4}$, Diane M. Renz ${ }^{1}$, Matthias Waginger ${ }^{1}$, Ekkehard Schleussner ${ }^{3}$, Dietmar

\begin{abstract}
Objective: Determine whether amniotic fluid volume (AFV) determination by magnetic resonance imaging (MRI) can be compared with ultrasound based amniotic fluid index (AFI) and whether diffusion weighted imaging (DWI) of the fetal kidneys can be used as surrogate marker for the assessment of AFV and fetal kidney function.

Methods: In the period from August 2013 to September 2014 prospective evaluation of AFV based on TRUFI MRI in 2 spatial planes (AFVMRI) and ultrasound based AFI. Inclusion criteria for the evaluation were: 1) Full image of the fetus (including AF and placenta) in an MR-study in at least two planes (sagittal and coronal), 2) Layer thickness $8 \mathrm{~mm}, 3$ ) DWI-images of the fetal kidney in two planes (sagittal and transverse to the fetus). ROI analysis of the ADC values in normal kidneys and in pathological fetal kidneys (CAKUT) as well as the correlation of AFVMRI vs. AFI, renal volume and ADC were performed.

Results: The correlation between AFI $(13.60 \pm 2.46 \mathrm{~cm})$ and AFVMRI $(579 \pm 353 \mathrm{~mL})$ was 0.69 ( $\mathrm{p}=0.03)$. There was no significant correlation between AFVMRI and kidney volume, between AFVMRI and ADC700 and ADC800. The B700-ADC values of fetuses with CAKUT (n=14) were considerably higher than the values in fetuses with healthy kidneys.

Conclusion: Compared to AFI, AFVMRI showed higher accuracy, but was associated with higher time consumption. Thus it cannot be routinely recommended for the purpose of AFV-determination alone. MRI can be used to establish a relationship between kidney diffusion parameters, renal volume, GA and AFV in fetuses with CAKUT.
\end{abstract}

\begin{abstract}
Abbreviations and symbols: ADC: apparent diffusion coefficient, AF: amniotic fluid, AFV: amniotic fluid volume, CAKUT: congenital anomalies of the kidney and urinary tract, DWI: diffusionweighted imaging, GA: gestational age, HASTE-T2: Half-Fourier Acquisition Single-shot Turbo spin-Echo, N: number, ROI-region of interest, SD: standard deviation, SDP: single deepest pocket, TOLAC: trial of labour after ceasarean section, TRUFI: True Fast Imaging with Steady State Free Precession, US: ultrasound, Wk: weeks
\end{abstract}

\section{Introduction}

The amniotic fluid (AF) plays an important role during pregnancy $[1,2]$. Its determination is an essential part of prenatal diagnostics [3]. Changes in the AFV are one of the most common abnormalities diagnosed by ultrasound (US). The amount of AF is assessed during US by measurement of the Amniotic Fluid Index (AFI) or the Single Deepest Pocket (SDP). Among other pathologies congenital anomalies of the kidney and urinary tract (CAKUT) are considered to have major influence on the amniotic fluid volume (AFV). They include a wide spectrum of renal abnormalities, from mild hydronephrosis to more severe cases, such as bilateral renal dysplasia. The etiology of the majority of cases of CAKUT remains unknown, but there is increasing evidence that genomic imbalance contributes to the pathogenesis of CAKUT [4].

For the accurate assessment of renal abnormalities, fetal MRI may be used as a further diagnostic step $[5,6]$. It not only determines some qualitative characteristics of the fetal kidney like the apparent diffusion coefficient (ADC) [7], but also provides a possibility for the determination of the AFV (AFVMRI). The study compared two methods of AF volumetry, and investigated the suitability of diffusionweighted imaging (DWI) of the fetal kidney to be used as a surrogate marker of fetal glomerular density in relation to the amount of AF.

Correspondence to: Elena Moschos, Institute of Diagnostic and Interventional Radiology, Section of Pediatric Radiology, University Hospital Jena, Germany, Tel: +49 36419324925; Fax: +49 36419324832; E-mail: elena.moschos@med.uni-jena.de

Key words: magnetic resonance imaging, amniotic fluid, kidney, diffusion magnetic resonance imaging, biomarkers

Received: April 28, 2017; Accepted: May 26, 2017; Published: May 29, 2017 


\section{Materials and methods}

\section{Subjects}

From August 2013 to September 2014 thirty pregnant women were investigated at a $1.5 \mathrm{~T}$ MRI scanner. The study protocol was approved by the local ethics committee. Informed consent was obtained from all prospectively recruited participants. All in the study included pregnant women underwent a medically indicated fetal MRI (Table 1).

\section{Study protocols}

MRI Protocol: All MR examinations were performed at $1.5 \mathrm{~T}$ (Magnetom Avanto, Siemens, Erlangen) using the standard body matrix coil and without application of contrast medium. All sequences listed below were included in the standard MRI protocol. They were obtained during free breathing and without sedation with the pregnant women in the supine or lateral position. For the determination of AFV a True Fast Imaging with Steady State Free Precession (TRUFI) gradient sequence was acquired in coronal and sagittal planes with the following parameters: TR $3.68 \mathrm{~ms}$; TE $1.84 \mathrm{~ms}$; slice thickness $8 \mathrm{~mm}$; gap $12 \mathrm{~mm}$ in 14 cases; no gap in 16 cases; acquisition time 71; (31 slices in the sagittal plane and 21 slices in the coronal plane).

T2-Half-Fourier Acquisition Single-shot Turbo spin-Echo (HASTE) sequences in the sagittal and axial plane with slice thickness varying from $2 \mathrm{~mm}$ to $6 \mathrm{~mm}$ were performed to determine the kidney volume, which was counted with the ellipsoid-formula (width $\mathrm{x}$ depth $\mathrm{x}$ length $\mathrm{x} 0.523 \mathrm{~mm}$ ) (Figure 1a, b).

Diffusion-weighted imaging: Diffusion parameters were evaluated with a Diffusion-Weighted-Sequence (TR 8000 ms; TE 90 ms; FOV 420 × 300 mm, matrix 192 × 192, slice thickness $8 \mathrm{~mm}$; Total

Table 1. Indications for fetal MRI.

\begin{tabular}{|c|c|c|}
\hline Number & & Indications \\
\hline 14 & CAKUT & $\begin{array}{l}\text { Congenital renal anomalies }(\mathrm{n}=2) \\
\text { Cystic dysplasia }(\mathrm{n}=1) \\
\text { Urinary tract disorder: Urinary tract diltation Grade I-III, } \\
\text { Hydronephrosis, Duplex kidney with Pyelectasia }(\mathrm{n}=11)\end{array}$ \\
\hline 8 & $\begin{array}{l}\text { CNS - } \\
\text { Malformation }\end{array}$ & $\begin{array}{l}\text { Arnold - Chiari }- \text { complex }(\mathrm{n}=1) \\
\text { Spina bifida }(\mathrm{n}=1) \\
\text { Posthemorrhagic hydrocephalus }(\mathrm{n}=1) \\
\text { Ventriculomegaly }(\mathrm{n}=3) \\
\text { Corpus callosum agenesis }(\mathrm{n}=1) \\
\text { Vermis cerebelli with fusion of the two cerebellar } \\
\text { hemispheres and enlarged Cisterna magna }(\mathrm{n}=1)\end{array}$ \\
\hline 1 & $\begin{array}{l}\text { Tuberous sclerosis } \\
\text { complex }\end{array}$ & \\
\hline 1 & $\begin{array}{l}\text { Planing trial } \\
\text { of labour after } \\
\text { ceasarean section } \\
\text { (TOLAC) }\end{array}$ & \\
\hline 1 & $\begin{array}{l}\text { Suspected } \\
\text { duodenal atresia }\end{array}$ & \\
\hline 1 & $\begin{array}{l}\text { Distended fetal } \\
\text { bowel loops }\end{array}$ & \\
\hline 1 & $\begin{array}{l}\text { Fetal: presacral } \\
\text { tumor mass } \\
\text { (anterior sacral } \\
\text { meningocele) }\end{array}$ & \\
\hline 1 & $\begin{array}{l}\text { Left-sided } \\
\text { diaphragmatic } \\
\text { hernia }\end{array}$ & \\
\hline 1 & $\begin{array}{l}\text { Fetal hydrops of } \\
\text { unknown origin }\end{array}$ & \\
\hline 1 & $\begin{array}{l}\text { Truncus arteriosus } \\
\text { communis }\end{array}$ & \\
\hline
\end{tabular}

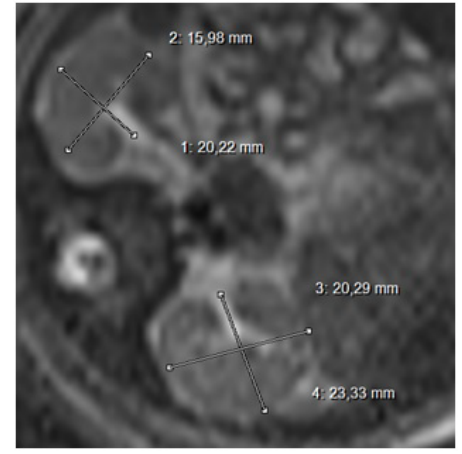

(a)

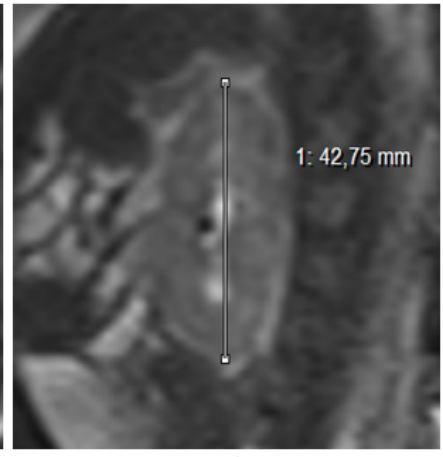

(b)
Figure 1. Measurements of the normal fetal kidney ( $34 \mathrm{wk})$ : a) width and depth in the axial plane, b) length in the sagittal plane in one of the two kidneys.

acquisition time $90 \mathrm{~s}$; b-factor $0,700 \mathrm{~s} / \mathrm{mm}^{2}$ or $0,400,800 \mathrm{~s} / \mathrm{mm}^{2}$ ). A total of 30 subjects were examined, of which 21 pregnancies were measured with a b-value of $700 \mathrm{~s} / \mathrm{mm}^{2}$, in 4 cases both b-values of 700 and of $800 \mathrm{~s} / \mathrm{mm}^{2}$ were sampled and in 5 cases b-value was $800 \mathrm{~s} / \mathrm{mm}^{2}$. Quantitative estimation of the ADC was by analyzing manually placed regions of interest (ROI). The best DW-Image of both fetal kidneys was selected and a $1 \mathrm{~cm}^{2}$ ROI was measured within the renal parenchyma in both kidneys in the transversal plane (Figure 2). The duration of the entire MR-examination including the time for patient preparation, coil placement and fetus localization in the data was approximately 30 minutes.

AF tracing: The AFV was evaluated on TRUFI sequences using the free open source software package 3D Slicer (Version 4.2.2.1, USA). The experience of the MRI operator was less than a year. The external and internal contours of the AF were marked manually for each layer both in the sagittal and coronal planes. Where possible, the umbilical cord was not included. The contoured area was filled with color (blue for AF) (Figure 3). The tracing of each study took approximately 40-60 minutes. For the overall volume calculation, the software interpolated the boundaries in these areas.

Fetal sonography: After the MRI scan, 16 from overall 30 subjects underwent transabdominal fetal sonography in a supine position with the devices GE Voluson E8 Expert (GE Healthcare), GE Voluson 730 Expert (GE Healthcare) or Toshiba Aplio 500 (Toshiba Medical Systems Corporation) using $3.5 \mathrm{MHz}$ curved-array transducers. The experience of the US operators was more than 5 years. The AFI measurement was performed similar to the method introduced initially by Phelan et al. [8] and described later by Hinh und Ladinsky [9]. The uterus was divided into four quadrants that are defined by the umbilicus. The transducer was placed vertically in each quadrant and then the largest fluid pocket in each quadrant was measured $(\mathrm{cm})$. To determine the AFI, the measurements from each quadrant were summed.

\section{Statistical analysis}

Statistical analysis was performed using SPSS 22.0 (IBM Corporation, US). Arithmetic mean values were calculated to describe the central tendency of a frequency distribution. The dispersion of a distribution in the subject groups was measured descriptively by calculating scattering measurements. To verify the correlation of MRtomographic volume measurements in both planes Bland-Altman plots were applied. The relationship between two characteristics in the subject groups was calculated by correlation analysis of bivariate statistics. In order to quantify the linear relationship in the subject groups, Pearson correlation coefficients were calculated. 


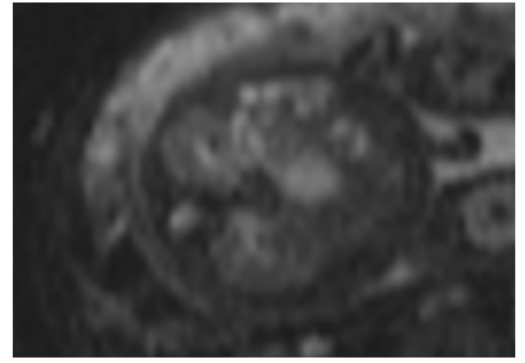

(a)

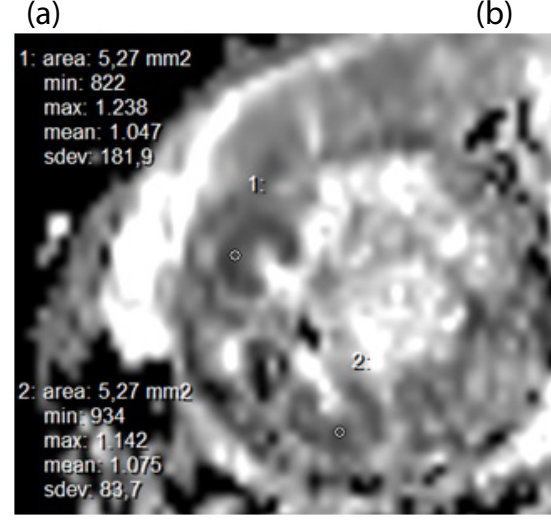

(c)

Figure 2. DWI-sequences (GA 34+3 wks): a) $b=0 \mathrm{~s} / \mathrm{mm}^{2}$, b) $b=700 \mathrm{~s} / \mathrm{mm}^{2}$ (trace image), c) ROI-placement and ADC-measurements with $\mathrm{b}=0,700 \mathrm{~s} / \mathrm{mm}^{2}$

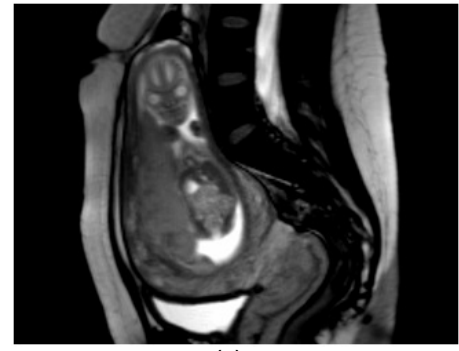

(a)

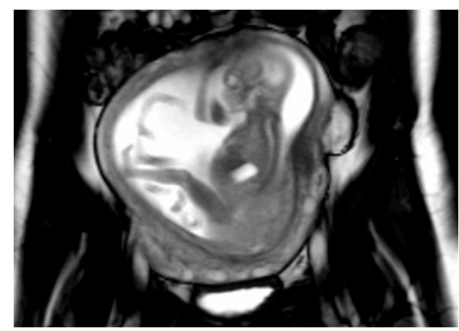

(c)

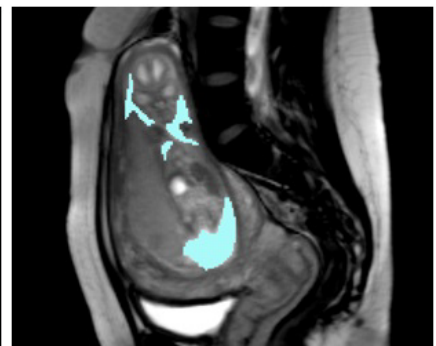

(b)

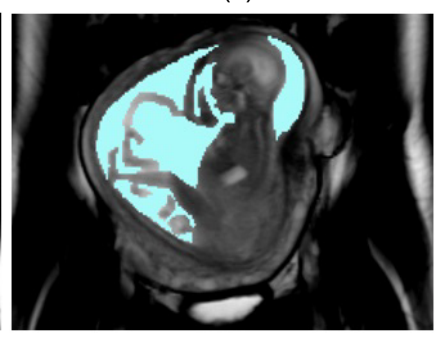

(d)
Figure 3. Before (a, c) and after (b, d) amniotic fluid tracing (blue) in a MR-dataset in two planes: a, b- sagittal towards the feet of the mother, c, d - coronar towards the feet of mother.

\section{Results}

In 30 cases the MRI was successfully performed. In $16 / 30$ cases [age $30 \mathrm{yrs}$ (21-39 yrs), gestational age (GA) 30 weeks (23-35wks)] the ultrasound evaluation of the AFV by AFI was performed on the same day with the MRI study (Group A). Other 14/30 patients underwent MRI alone without AFI-measurement (Group B). The GA in the Group B was 26 weeks and 2 days (19-33 wks), the age of the pregnant women 26.2 years (23-36 yrs).

The mean AFI in the Group A was $13.6 \pm 2.46 \mathrm{~cm}$ (range $8.30-$ $17.20 \mathrm{~cm}$ ). The AFVMRI was measured in a total of 30 patients in both groups. The mean AFVMRI was $579 \pm 353 \mathrm{~mL}$ (range 156-1732 mL) in coronal and $590 \pm 335 \mathrm{~mL}$ (range 187-1628 ml) in sagittal plane (Figure 4).

The mean difference of AFVMRI (coronal-sagittal) was $-5.09 \mathrm{~mL}$ (SD $36 \mathrm{ml}$ ), the upper and lower limits of agreement were $67 \mathrm{~mL}$ and -77 $\mathrm{mL}$, respectively. The correlation between AFI and AFVMRI was 0.69 $(p=0.03)$; there was a linear relationship between MR-tomographic and sonographic determined values (Figure 5 ). The range of renal volume obtained was $0.57-21.92 \mathrm{~mL}$ (Table 2).

Inclusion criteria for the evaluation were: 1) Full image of the fetus (including $\mathrm{AF}$ and placenta) in an MR-study in at least two planes (sagittal and coronal), 2) Layer thickness $8 \mathrm{~mm}, 3$ ) DWI-images of the fetal kidney in two planes (sagittal and transverse to the fetus). 16 patients underwent MRI followed by the US evaluation of the AFI on the same day (group A). The other 14/30 patients underwent MRI alone without AFI-measurement (group B). Exclusion criteria for the group A were absence of AFI on the same day and for both groups inadequate image quality, absence of DWI of TRUFI sequences as well as poor orientation of the fetal kidneys in very small/rolled up fetuses.

The diffusion parameters of the fetal kidneys were measured in 30 fetuses. ADC-values of the fetal kidneys determined in 25 patients with a b-value of $700 \mathrm{~s} / \mathrm{mm}^{2}$ were on average lower (mean $1.5 \times 10^{-3}$, min 0.67 , max 2.99, SD 0.53) than in the group of 9 pregnancies measured with a b-value of $800 \mathrm{~s} / \mathrm{mm}^{2}$ (mean $1.58 \times 10^{-3}$, min 1.22, $\max 2.74$, SD $0.47)$. The average value of ROI-size was $10 \mathrm{~mm}\left(\mathrm{SD} \pm 6.69 \mathrm{~mm}^{2}\right)$.

The comparison of ADC values in fetuses with and without CAKUT showed that on b-value of $700 \mathrm{~s} / \mathrm{mm}^{2}$ the ADC was higher than in fetal kidneys without anomalies (Table 3).

The Pearson correlation coefficients between AFV measured both in MRI and in the sonography and diffusion parameters of the fetal kidneys for fetuses with CAKUT are summarized in Table 4. There was no significant correlation between diffusivity of the fetal kidney and with both methods measured AFV in fetuses with CAKUT ( $>0.05)$.

Table 2. MR-tomographically measured kidney volume $(n=30)$.

\begin{tabular}{|c|c|c|c|c|}
\hline $\begin{array}{c}\text { Kidney volume } \\
(\mathrm{mL})\end{array}$ & $\mathrm{N}$ & Minimum & Maximum & $\mathrm{SD}$ \\
\hline KVol_right & 29 & 0.57 & 21.92 & 4.84 \\
\hline KVol_left & 29 & 0.57 & 20.50 & 6.13 \\
\hline
\end{tabular}

Table 3. Diffusion values $\mathrm{b}=700 \mathrm{~s} / \mathrm{mm}^{2} \mathrm{ADC}$ and $\mathrm{b}=800 \mathrm{~s} / \mathrm{mm}^{2}$; ADC in the overall group in healthy kidneys fetuses $(n=16)$ and fetuses with CAKUT $(n=14)$.

\begin{tabular}{|c|c|c|}
\hline $\begin{array}{c}\text { Mean Diffusion } \\
\left(\mathrm{mm}^{2} / \mathrm{s}\right)\end{array}$ & CAKUT $(\mathrm{n}=14)$ & $\begin{array}{c}\text { Healthy kidneys prenatal } \\
(\mathrm{n}=16)\end{array}$ \\
\hline & $1.03-2.99 \times 10-^{3}(\mathrm{n}=14)$ & $0.67-2.14 \times 10-^{3}(\mathrm{n}=11)$ \\
B700ADC & Mean $1.64 \times 10-^{-3}$ & Mean $1.34 \times 10-^{3}$ \\
& SD $0.61 \times 10-^{-3}$ & SD $0.37 \times 10-^{-3}$ \\
\hline & $1.30-1.75 \times 10-^{3}(\mathrm{n}=3)$ & $1.22-2.74 \times 10-^{3}(\mathrm{n}=6)$ \\
B800ADC & Mean $1.56 \times 10-^{-3}$ & Mean $1.59 \times 10-^{3}$ \\
& SD $0.23 \times 10-^{-3}$ & SD $0.58 \times 10-^{3}$ \\
\hline
\end{tabular}

Table 4. Pearson correlation between the AFV measured in MRI and in the sonography and kidney - diffusivity (ADC) of different b-values in fetuses with CAKUT: $b=700 \mathrm{~s} / \mathrm{mm}^{2}$ and $\mathrm{b}=800 \mathrm{~s} / \mathrm{mm}^{2}$

\begin{tabular}{|c|c|c|c|c|}
\hline \multirow{2}{*}{ Parameter } & \multicolumn{2}{|c|}{ AFVMRI $(\mathrm{mL})$} & \multicolumn{2}{c|}{ AFI (cm) } \\
\cline { 2 - 5 } & $\mathrm{B} 700 \mathrm{ADC}\left(\mathrm{mm}^{2} / \mathrm{s}\right)$ & $\mathrm{B} 800 \mathrm{ADC}\left(\mathrm{mm}^{2} / \mathrm{s}\right)$ & $\mathrm{B} 700\left(\mathrm{~mm}^{2} / \mathrm{s}\right)$ & $\mathrm{B} 800\left(\mathrm{~mm}^{2} / \mathrm{s}\right)$ \\
\hline $\begin{array}{c}\text { Pearson- } \\
\text { correlation }\end{array}$ & -0.218 & -0.594 & 0.522 & -0.558 \\
\hline Sig. & 0.546 & 0.214 & 0.229 & 0.623 \\
\hline $\mathrm{N}$ & 10 & 6 & 7 & 3 \\
\hline
\end{tabular}




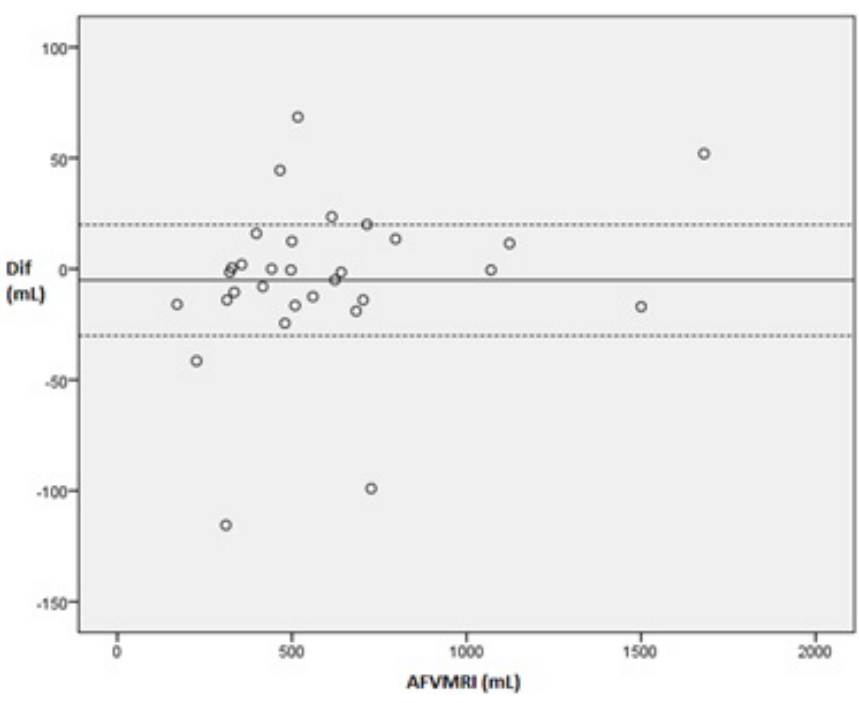

Figure 4. Bland-Altman-plot of MR-tomographic amniotic fluid volume measurement in coronar and sagittal plane $(n=30)$.

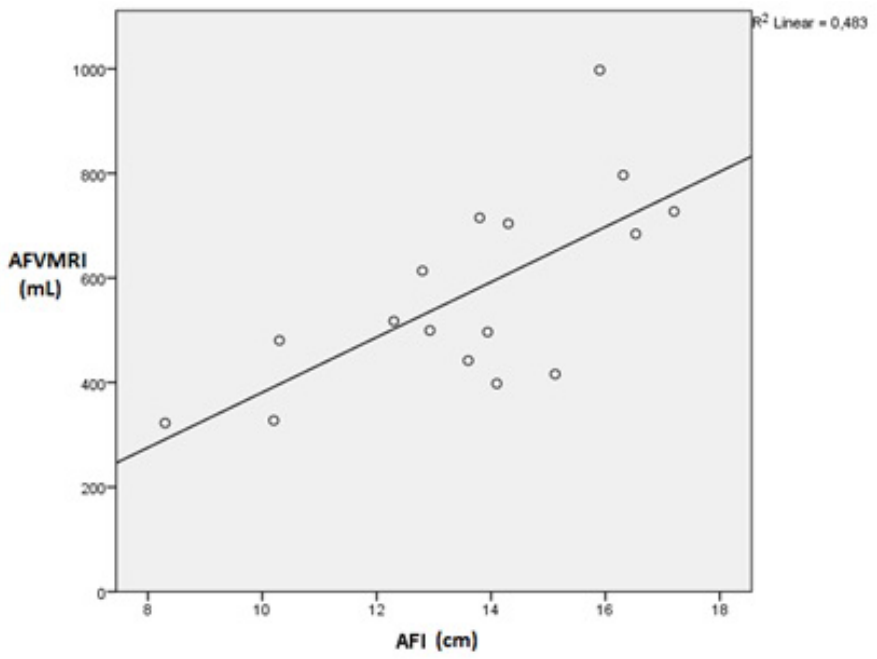

Figure 5. Scatter graph of the correlation of AFI and AFVMRI $(n=16)$

For fetuses with healthy kidneys the correlation data between AFV and kidney ADC was not obtained because in that group 15 fetuses presented with pathologies which can affect the AFV regardless of kidney function (Table 1).

Renal diffusion parameters in both groups were correlated to GA. With increasing GA the ADC-values in the fetal kidney decreased (Pearson correlation coefficient of $-0.653(p<0.01)$ when measured with b-value of $700 \mathrm{~s} / \mathrm{mm}^{2}$ ). When measured with b-value of $800 \mathrm{~s} / \mathrm{mm}^{2}$ the data was not statistically significant (Pearson correlation coefficient of $-0.465(p=0.207)$ ), which is probably due to a fewer number of patients in that group.

A correlation between the kidney diffusion parameters and total kidney volume measured in the MRI was also performed (Table 5).

The negative correlation in the Table 5 was statistically significant when measured with $b$-value of $700 \mathrm{~s} / \mathrm{mm}^{2}(\mathrm{p}<0.05)$, suggesting that fetuses with reduced renal volume show higher ADC-values. This finding is in concordance with a physiological increase in kidney volume with increasing GA (Figure 6).
The measured kidney volume in both groups correlated significantly with the GA with Pearson correlation coefficient of $0.673(\mathrm{p}=0)$ (Figure 6)

Two outliers were observed. They are marked in red and blue, respectively. They are accounting for one case of severe subvesical obstruction with megacystitis and enlargement of both ureters and the renal pelvis (red) and one case of bilateral pyelectasia (blue) in the fetal MRI which consequently lead to an increased kidney volume since the parenchyma could not be evaluated separately.

\section{Discussion}

\section{AFV measurements}

Fetal MRI allows measurement of AFV. AFVMRI in two planes are only very slightly different and show low SD based on the orientation. This indicates that in the future in order to determine the AFVMRI one plane will be sufficient enough.

The TRUFI sequences were selected for the AFV evaluation in the MRI. Due to the high difference in contrast, they allow a good differentiation between the bright water and other structures (fetus, placenta, uterus, umbilical cord). They can be performed with free breathing, are rapidly acquirable and have a high signal - to - noise ratio [10]. TRUFI sequences in the group B were performed with intersection gap of $12 \mathrm{~mm}$, which could lead to partial volume effect [11]. In order to avoid it, achieve better image quality and thus more exact AFV evaluation TRUFI sequences in the group A were performed without intersection gap.

In the current study the manual AFV tracing has been performed. None the less, some studies on the semiautomatic MR volumetry of fetal organs, such as the fetal brain and the fetal lung have already been published $[12,13]$. The semitomatic method is time-saving [14] However, AF tracing in an MR dataset, in contrast to, for example, lung

Table 5: Pearson correlation between the kidney volume measured in MRI and kidney diffusivity (ADC) of different b-values: $\mathrm{b}=700 \mathrm{~s} / \mathrm{mm}^{2}$ and $\mathrm{b}=800 \mathrm{~s} / \mathrm{mm}^{2}$.

\begin{tabular}{|c|c|c|}
\hline Kidney volume $(\mathrm{mL})$ & $\begin{array}{c}\mathrm{B} 700 \mathrm{ADC} \\
\left(\mathrm{mm}^{2} / \mathrm{s}\right)\end{array}$ & $\begin{array}{c}\mathrm{B} 800 \mathrm{ADC} \\
\left(\mathrm{mm}^{2} / \mathrm{s}\right)\end{array}$ \\
\hline KVol & -0.416 & -0.513 \\
\hline Sig. & 0.043 & 0.158 \\
\hline $\mathrm{N}$ & 24 & 9 \\
\hline
\end{tabular}

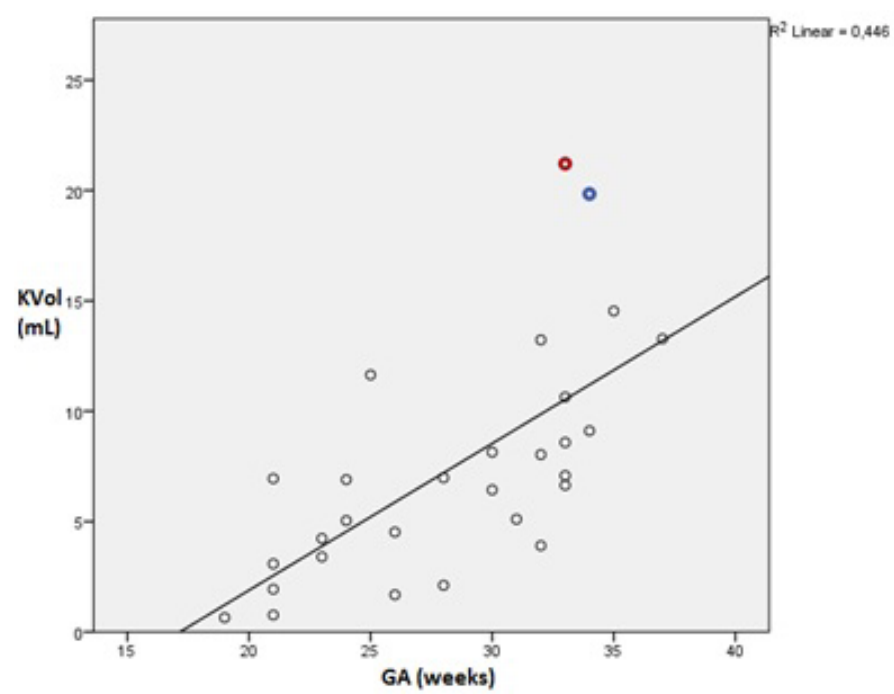

Figure 6. Scatter plot of correlation between gestational age and kidney volume in both groups $(\mathrm{n}=30)$. 
tracing, is a more difficult task since the AF does not possess a clearly defined shape. For this reason, no semiautomatic method was used in the present study.

The AFI-value as an equivalent of an AFV ranged from 8.30 to $17.20 \mathrm{~cm}$ with a mean of $13.60 \mathrm{~cm}$ (SD 2.46). The mean AFI of our study is slightly higher than that of Magann et al. [15], where it was 10.4 $\pm 4.2 \mathrm{~cm}$. Nevertheless, that study [15] also included early pregnancies (from 14 weeks), while our study involved pregnancies from $19^{\text {th }}$ week onwards. The AFI-range in the current study is somewhat lower than that of Hebbar et al. [16], where the range was from 11.7 to $17.3 \mathrm{~cm}$. This can be explained by the fact that it included women from 34 to 40 GA weeks and also multiple pregnancies. When comparing the results of our study with other data, a strong correlation between AFI and patient population or GA can be followed.

There was a statistically significant correlation between AFVMRI and AFI. The comparative study of Zaretsky et al. [17] with a similar design did not demonstrate a direct comparison between MRtomographic and sonographic method of AF volumetry. However, it showed a correlation between AFI and AFVMRI, measured 3 hours before the Caesarean section and the AFV collected intrapartum during the Caesarean section: AFVMRI compared to AFI correlated higher with the measured during Cesarean section AFV [17]. That study [17] also showed that AFI sensitivity declined with decreasing AFV in oligohydramnios. In the current study AFI variation between US and MRI did not change the categorization between normal AFV, reduced AFV or polyhydramnios. Another earlier study showed that AFI underestimated the AFV in oligohydramnios which consequently could lead to unnecessary labor induction [18]. The reason for this is most likely the inaccuracy of the volume estimation with the AFI, which is also confirmed by the low reproducibility of the measurement in pregnancies with low AFV [19]. That is why the application of the Single Deepest Pocket (SDP) in oligohydramnios is currently preferred [20].

Even though the use of fetal MRI to determine the amount of AF instead of the established sonographic method (AFI), is associated with high exactness of MRI, it brings a lot of methodical disadvantages of MRI over sonography. These include basic limitations of MRI such as a sufficient diagnostic image quality and absence of contraindications for the MR-study. Logistic time requirement for the fetal MR-study as well its cost-effectiveness do not allow MRI to be routinely recommended for the purpose of AFV determination alone. It could be however useful in patients with sonogaphically diagnosed changes in the AFV. So, both in severe oligo- and polyhydramnios,, it may complement to identify the cause of AFV-change and determine pregnancy outcome [21].

\section{Renal imaging}

In the current study the kidney volume was calculated using the formula for an ellipsoid in order to achieve a more exact comparison with the published size and volume charts [22]. However, the study of Bakker et al. [23] showed that for reliable calculation of renal size in vitro, MRI with use of the voxel-count method is preferred. Compared to the study of Michielsen et al. [2], where the MR-volumetry was performed in 240 fetuses, the range of the kidney volumes in the current study was slightly larger (Table 2). That study [2] however, included only healthy fetal kidneys, while our study involved also fetuses with CAKUT and pregnancies with earlier GA. In fetuses with CAKUT renal size is not an accurate reflect of the renal function (Beall et al. 2007). Moreover, the exact renal volume estimation in the fetal MRI using diameter measurement is only possible to a limited extent due to the small kidney size. Even though the present study allows a rough definition of the kidneys in the DWI and in the TRUFI sequences, for a volumetry a spatially higher resolution sequence (at best a 3D sequence), would have been necessary. This was not possible in the context of this study because of required longer time consumption for each MRI scan.

In most pregnancies $(n=25)$ the diffusivity was measured with $a b$ - value of $700 \mathrm{~s} / \mathrm{mm}^{2}$. It was with $1.50 \times 10^{3} \mathrm{~mm}^{2} / \mathrm{s}$ on average slightly smaller than that of a b-value of $800 \mathrm{~s} / \mathrm{mm}^{2}$ (Table 3).

The other studies in which the fetal renal diffusivity was the subject of the study were performed with maximum b values of $700 \mathrm{~s} / \mathrm{mm}^{2}$ and minimum $b$ values of $0,50,200$ and $350 \mathrm{~s} / \mathrm{mm}^{2}$, respectively [7,24-26]. With increasing $b$-value the signal-to-noise ratio in the image reduces and thereby the sensitivity to motion and to other artifacts increases $[27,28]$. Therefore, it is most likely that a signal-bias in the current study was responsible for small increase in diffusivity with $b$-values of $800 \mathrm{~s} / \mathrm{mm}^{2}$.

We observed a significant negative correlation between the diffusivity of the renal parenchyma and the fetal kidney volume $(\mathrm{p}<0.05)$ (Table 5). It was expected because it is known that with increasing GA the glomerular density increases (24). This leads to increase in the renal volume, kidney density, isotropy in the kidney and consequently to the restriction in the diffusion parameters in the parenchyma $[29,30]$.

The study showed no statistically significant correlation between the AFVMRI/AFI and kidney diffusion when measured with b-values of 700 and $800 \mathrm{~s} / \mathrm{mm}^{2}$ in fetuses with CAKUT. Another study of 38 fetuses with hypoplastic kidneys demonstrated that in $88 \%$ of cases they developed oligohydramnios [31]. The reason for the lack of significance between fetal kidney diffusion values and AFVMRI is probably due to the relatively small number of pregnancies in the study.

The diffusivity of the kidneys in fetuses with CAKUT was higher than the diffusivity of healthy fetal kidneys. The study of Chaumoitre et al. [7] showed that of total 6 fetuses ( 3 fetuses with nephropathy and 3 fetuses with dilation of the urinary system) 4 fetuses had increased ADC-values with a b-value of $300 \mathrm{~s} / \mathrm{mm}^{2}$. In contrast, in another study of Savelli et al. [25], the diffusivity in 15 fetuses with CAKUT was slightly lower than in fetuses with normal renal function $\left(\mathrm{b}\right.$-value of $700 \mathrm{~s} / \mathrm{mm}^{2}$ ). The increase in diffusion values in fetuses with CAKUT is possibly caused by changes in the glomerular density in pathological renal kidneys compared to healthy fetal kidneys. To the best of our knowledge there are currently no fetal MR-studies that have investigated these changes in fetuses with CAKUT. However, some studies on diffusivity in renal pathology in adults and children show that the diffusivity of the renal parenchyma in newborns with congenital hydronephrosis is lower than of those fetuses with healthy kidneys [32]. ADC-values of healthy fetuses are lower than in newborns [24,32] which can be explained by the fact that the renal perfusion and the GFR are lower in fetuses and increase after birth [30], while the glomerular density is already determined at birth and thus has no influence on the diffusivity. In this study, it was shown that there is a negative correlation between the ADC-values with b-values of 700 and $800 \mathrm{~s} /$ $\mathrm{mm}^{2}$ and the GA, which was significant for a b-value of $700 \mathrm{~s} / \mathrm{mm}^{2}$. This result is consistent with data of other studies $[7,24,26]$, which indicates that the fetal kidney diffusion values are generally well reproducible. We suggest that an ADC may possibly serve as an indicator of the fetal renal function. In adults, the diffusion value is already successfully used for this purpose $[9,33,34]$.

\section{Limitations of the study}

The study was based on the prenatal diagnostics and it did not evaluate the fetal outcome postnatal. Further studies such as follow up of 
the CAKUT fetuses are necessary in order to confirm the prenatal data and prove the concept of the glomerular density. Some of the fetuses which demonstrated no renal abnormalities on US or fetal MRI, could still have renal impairment due to their syndromes and conditions, for example those with tuberous sclerosis. Thus introduction of more homogenous healthy renal comparison group could reinforce the study outcome.

\section{Conclusion}

AFV measurement with both MRI and US demonstrated a significant correlation between the two methods. Although AFVMRI, compared to the sonographic AFI, showed higher accuracy, this method was associated with significantly higher time consumption. For this reason, it cannot be routinely recommended for the purpose of AFV-determination alone. One can suspect an advantage of the AFVMRI in poly- and oligohydramnios in a way that it may help to determine the underlying cause and thus pregnancy outcome. The role of AFVMRI in the fetuses without CAKUT was not explored in the current study. For this purpose, more intensive studies with larger study populations are needed.

We suggest that MRI can be used in order to establish a relationship between kidney diffusion parameters, renal volume, GA and AFV in fetuses with CAKUT. The MR-tomographically determined diffusivity can possibly serve as maturation marker, which can in accordance to GA differentiate between 'healthy' and 'diseased' fetal kidneys. More extensive research is required to secure the connection between fetal diffusivity and kidney function in order to make it prognostically relevant.

\section{Authorship and contributorship}

Byline authors: Hans-Joachim Mentzel, Daniel Güllmar, Uwe Schneider, Anja Fiedler, Dietmar Schlembach, Elena Moschos.

Contributors: Ulrike John, Diane M. Renz, Matthias Waginger, Ekkehard Schleussner.

\section{Acknowledgements}

This research was supported by the Department of Obstetrics and Institute of Diagnostic and Interventional Radiolgy of the University Hospital Jena. We are thankful to our colleagues PD Dr. Ulrike John, PD Dr. Diane M. Renz, Dr. Matthias Waginger who provided expertise that greatly assisted the research. We are also grateful to Prof. Dr. Ekkehard Schleussner for making substantial contribution to conception of the study.

\section{References}

1. Moore TR (2011) The role of amniotic fluid assessment in evaluating fetal well-being. Clinics in perinatology 38: 33-46.

2. Michielsen K, Meersschaert J, De Keyzer F, Cannie M, Deprest J et al. (2010) MR volumetry of the normal fetal kidney: reference values. Prenatal diagnosis 30: 1044-8.

3. Nabhan AF, Abdelmoula YA (2008) Amniotic fluid index versus single deepest vertical pocket as a screening test for preventing adverse pregnancy outcome. Cochrane Database Syst Rev 16: CD006593 [Crossref]

4. Uy N, Reidy K (2016) Developmental Genetics and Congenital Anomalies of the Kidney and Urinary Tract. J Pediatr Genet 5: 51-60. [Crossref]

5. Caire JT, Ramus RM, Magee KP, Fullington BK, Ewalt DH et al. (2003) MRI of fetal genitourinary anomalies. AJR American Journal of Roentgenology 181: 1381-5.

6. Pico H, Dabadie A, Bourliere-Najean B, Philip N, Capelle M, et al. (2014) Contribution of the foetal uro-MRI in the prenatal diagnosis of uronephropathies. Diagn Interv Imaging 95: 573-578. [Crossref]
7. Chaumoitre K, Colavolpe N, Shojai R, Sarran A, D'Ercole, et al. (2007) Diffusionweighted magnetic resonance imaging with apparent diffusion coefficient (ADC) determination in normal and pathological fetal kidneys. Ultrasound Obstet Gynecol 29: 22-31. [Crossref]

8. Phelan JP1, Ahn MO, Smith CV, Rutherford SE, Anderson E (1987) Amniotic fluid index measurements during pregnancy. J Reprod Med 32: 601-604. [Crossref]

9. Hinh ND, Ladinsky JL (2005) Amniotic fluid index measurements in normal pregnancy after 28 gestational weeks. International journal of gynaecology and obstetrics: the official organ of the International Federation of Gynaecology and Obstetrics 91: 132-6.

10. Chung HW, Chen CY, Zimmerman RA, Lee KW, Lee CC, et al. (2000) T2-Weighted fast MR imaging with true FISP versus HASTE: comparative efficacy in the evaluation of normal fetal brain maturation. AJR American Journal of Roentgenology 175: 1375-80.

11. Gonzalez Ballester MA, Zisserman AP, Brady M (2002) Estimation of the partial volume effect in MRI. Medical image analysis. Med Image Anal 6: 389-405. [Crossref]

12. Berger-Kulemann V, Berger R, Mlczoch E, et al (2015) The Effects of Hemodynamic Alterations on Lung Volumes in Fetuses with Tetralogy of Fallot: An MRI Study. Pediatr Cardiol 36: 1287-93. [Crossref]

13. Rubesova E1 (2016) Why do we need more data on MR volumetric measurements of the fetal lung? Pediatr Radiol 46: 167-171. [Crossref]

14. Gholipour A, Estroff JA, Barnewolt CE, Connolly SA, Warfield SK (2011) Fetal brain volumetry through MRI volumetric reconstruction and segmentation. Int $J$ Comput Assist Radiol Surg 6: 329-39. [Crossref]

15. Magann EF, Sanderson M, Martin JN, Chauhan S (2000) The amniotic fluid index, single deepest pocket, and two-diameter pocket in normal human pregnancy. $\mathrm{Am} J$ Obstet Gynecol 182: 1581-7. [Crossref]

16. Hebbar S, Rai L, Adiga P, Guruvare S (2015) Reference ranges of amniotic fluid index in late third trimester of pregnancy: what should the optimal interval between two ultrasound examinations be? J Pregnanc 319204.

17. Zaretsky MV1, McIntire DD, Reichel TF, Twickler DM (2004) Correlation of measured amnionic fluid volume to sonographic and magnetic resonance predictions. Am J Obstet Gynecol 191: 2148-2153. [Crossref]

18. Moore TR1 (1990) Superiority of the four-quadrant sum over the single-deepest-pocket technique in ultrasonographic identification of abnormal amniotic fluid volumes. $\mathrm{Am} \mathrm{J}$ Obstet Gynecol 163: 762-767. [Crossref]

19. Magann EF1, Chauhan SP, Barrilleaux PS, Whitworth NS, Martin JN (2000) Amniotic fluid index and single deepest pocket: weak indicators of abnormal amniotic volumes. Obstet Gynecol 96: 737-740. [Crossref]

20. Magann EF1, Chauhan SP, Doherty DA, Magann MI, Morrison JC (2007) The evidence for abandoning the amniotic fluid index in favor of the single deepest pocket. Am J Perinatol 24: 549-555. [Crossref]

21. Sohn YS, Kim MJ, Kwon JY, Kim YH, Park YW (2007) The usefulness of fetal MRI for prenatal diagnosis. Yonsei Med J 48: 671-7. [Crossref]

22. van Vuuren SH, Damen-Elias HA, Stigter RH, et al (2012) Size and volume charts of fetal kidney, renal pelvis and adrenal gland. Ultrasound Obstet Gynecol 40: 659-64. [Crossref]

23. Bakker J, Olree M, Kaatee R, de Lange EE, Beek FJ (1998) In vitro measurement of kidney size: comparison of ultrasonography and MRI. Ultrasound Med Biol 24: 683-8. [Crossref]

24. Witzani L, Brugger PC, Hormann M, Kasprian G, Csapone-Balassy C (2006). Normal renal development investigated with fetal MRI. European Journal of Radiology 57: 294-302.

25. Savelli S, Di Maurizio M, Perrone A, et al (2007) MRI with diffusion-weighted imaging (DWI) and apparent diffusion coefficient (ADC) assessment in the evaluation of normal and abnormal fetal kidneys: preliminary experience. Prenatal Diagnosis 27: 1104-11. [Crossref]

26. Manganaro L, Francioso A, Savelli S, et al (2009) Fetal MRI with diffusion-weighted imaging (DWI) and apparent diffusion coefficient (ADC) assessment in the evaluation of renal development: preliminary experience in normal kidneys. La Radiologia Medica 114: 403-13.

27. Colagrande S, Belli G, Politi LS, Mannelli L, Pasquinelli F, Villari N (2008) The influence of diffusion- and relaxation-related factors on signal intensity: an introductive guide to magnetic resonance diffusion-weighted imaging studies. Journal of Computer Assisted Tomography 32: 463-74. 
28. Battal B1, Akg ̃̃1/4n V, KocaoÄŸlu M (2012) Diffusion-weighted MRI beyond the central nervous system in children. Diagn Interv Radiol 18: 288-297. [Crossref]

29. Ebrahimi B, Textor SC, Lerman LO (2014) Renal relevant radiology: renal functional magnetic resonance imaging. Clin J Am Soc Nephrol 9: 395-405. [Crossref]

30. Jones RA1, Grattan-Smith JD (2003) Age dependence of the renal apparent diffusion coefficient in children. Pediatr Radiol 33: 850-854. [Crossref]

31. Spaggiari E, Stirnemann JJ, Heidet L, et al.2013. Outcome following prenatal diagnosis of severe bilateral renal hypoplasia. Prenatal Diagnosis 33: 1167-72.
32. Lin F, Li Z, Gan Y, et al (2014) Relationship between renal apparent diffusion coefficient values and glomerular filtration rate in infants with congenital hydronephrosis. Biosci Trends 8: 274-279. [Crossref]

33. Fan WJ, Ren T, Li Q, et al (2015) Assessment of renal allograft function early after transplantation with isotropic resolution diffusion tensor imaging. European radiology 26: 567-575.

34. Goyal A1, Sharma R, Bhalla AS, Gamanagatti S, Seth A (2012) Diffusion-weighted MRI in assessment of renal dysfunction. Indian J Radiol Imaging 22: 155-159. [Crossref]

Copyright: $(02017$ Moschos E. This is an open-access article distributed under the terms of the Creative Commons Attribution License, which permits unrestricted use, distribution, and reproduction in any medium, provided the original author and source are credited. 